\title{
Commutativity of Prime Gamma Rings with Left Centralizers
}

\author{
K. K. Dey and A. C. Paul \\ Department of Mathematics, Rajshahi University, Rajshahi-6205, Bangladesh \\ Received 14 May 2013, accepted in final revised form 26 November 2013
}

\begin{abstract}
Let $M$ be a $\Gamma$-ring. If $M$ satisfies the condition (*) $x \alpha y \beta z=x \beta y \alpha z$ for all $x, y, z \in M, \alpha, \beta \in \Gamma$, then we investigate commutativity of prime $\Gamma$-rings satisfying certain identities involving left centralizer.

Keywords: Prime $\Gamma$-ring; Derivation; Generalized derivation; Left centralizer.

() 2014 JSR Publications. ISSN: 2070-0237 (Print); 2070-0245 (Online). All rights reserved. doi: http://dx.doi.org/10.3329/jsr.v6i1.14872 $\quad$ J. Sci. Res. 6 (1), 69-77 (2014)
\end{abstract}

\section{Introduction}

Let $M$ and $\Gamma$ be additive abelian groups. If there exists a mapping of $M \times \Gamma \times M \rightarrow M$ which satisfies the conditions:

(i) $(x+y) \alpha z=x \alpha z+y \alpha z, x(\alpha+\beta) y=x \alpha y+x \beta y$, $x \alpha(y+z)=x \alpha y+x \alpha z$

(ii) $(x \alpha y) \beta z=x \alpha(y \beta z)$ for all $x, y, z \in M$ and $\alpha, \beta \in \Gamma$, then $M$ is called a $\Gamma$-ring in the sense of Barnes [1].

Throughout this paper $M$ denotes a $\Gamma$-ring with center $Z(M)$. For any $x, y \in M, \alpha \in \Gamma$, the symbol $[x, y]_{\alpha}$ (resp. $\langle x, y\rangle_{\alpha}$ will denote the commutator $x \alpha y-y \alpha x$ (resp. the anticommutator $x \alpha y+y \alpha x)$. The symbol $[\alpha, \beta]_{x}$ is defined by $\alpha x \beta-\beta x \alpha$ for all $x \in M, \alpha, \beta \in \Gamma$. A $\Gamma$-ring $M$ is called commutative if $[x, y]_{\alpha}=0$ for all $x, y \in M, \alpha \in \Gamma$. We know that

$[x \beta y, z]_{\alpha}=[x, z]_{\alpha} \beta y+x \beta[y, z]_{\alpha}+x[\beta, \alpha]_{z y}$ and

$[x, y \beta z]_{\alpha}=y \beta[x, z]_{\alpha}+[x, y]_{\alpha} \beta z+y[\beta, \alpha]_{x z}$.

We take an assumption (*) $x \beta z \alpha y=x \alpha z \beta y$ for all $x, y, z \in M, \alpha, \beta \in \Gamma$. Using the assumption the basic commutator identities reduce to 
$[x \beta y, z]_{\alpha}=[x, z]_{\alpha} \beta y+x \beta[y, z]_{\alpha}$ and

$[x, y \beta z]_{\alpha}=y \beta[x, z]_{\alpha}+[x, y]_{\alpha} \beta z$.

We shall make extensive use of the following basic commutator identities throughout the discussion without any specific mention:

$$
\begin{aligned}
& \langle x, y \beta z\rangle_{\alpha}=\langle x, y\rangle_{\alpha} \beta z-y \beta[x, z]_{\alpha}=y \beta\langle x, z\rangle_{\alpha}+[x, y]_{\alpha} \beta z, \\
& \langle x \beta y, z\rangle_{\alpha}=x \beta\langle y, z\rangle_{\alpha}-[x, z]_{\alpha} \beta y=\langle x, z\rangle_{\alpha} \beta y+x \beta[y, z]_{\alpha} .
\end{aligned}
$$

Recall that $M$ is prime if $x \Gamma M \Gamma y=\{0\}$ implies that $x=0$ or $y=0$. An additive mapping $T: M \rightarrow M$ is called a left (resp. right) centralizer if $T(x \alpha y)=T(x) \alpha y$ (resp. $T(x \alpha y)=x \alpha T(y))$, holds for all $x, y \in M, \alpha \in \Gamma$. A multiplier is an additive mapping which is both right as well as left centralizer. An additive mapping $d: M \rightarrow M$ is called a derivation if $d(x \alpha y)=d(x) \alpha y+x \alpha d(y)$, holds for all $x, y \in M, \alpha \in \Gamma$. An additive mapping $d: M \rightarrow M$ is said to be a left derivation if $d(x \alpha y)=x \alpha d(y)+y \alpha d(x)$ holds for all $x, y \in M, \alpha \in \Gamma$. An additive mapping $D: M \rightarrow M$ is said to be a generalized derivation on $M$ if there exists a derivation $d: M \rightarrow M$ such that $D(x \alpha y)=D(x) \alpha y+x \alpha d(y)$ holds for all $x, y \in M, \alpha \in \Gamma$. Obviously, generalized derivation with $d=0$, covers the concept of left centralizers.

Many authors have investigated the relationship between the commutativity of the classical rings and certain types of derivations. In this direction, the first result was established by Posner [2]. Here he proved that if a prime ring $R$ admits a nonzero derivation $d$ such that $[d(x), x] \in Z(R)$ for all $x \in R$, then $R$ is commutative. Afterwards, many algebraists refined and extended the Posner's result. In [3], Bresar and Vukman proved that a prime ring $R$ admits a nonzero left derivation, then $R$ is commutative. Recently, many authors have obtained commutativity results for prime and semiprime rings with derivation, left derivation and generalized derivation.

In ref. [4], Asci and Ceran investigated commutativity of prime $\Gamma$-rings with left and right derivations. Afterwards, Soyturk [5] obtained the commutativity in prime $\Gamma$-rings with derivations. In [6], Hoque and Paul worked on centralizers of semiprime $\Gamma$-rings and proved that under certain condition, every Jordan left centralizer is a left centralizer. They also proved that every Jordan centralizer is a centralizer. Dey and Paul [7] proved that a mapping $T: M \rightarrow M$ is a centralizer if and only if it is centralizing left centralizer. They also showed that if $T$ and $S$ are left centralizers of $M$ in which $T(x) \alpha x+x \alpha S(x) \in Z(M)$ for all $x \in M$, and $\alpha \in \Gamma$, then both $T$ and $S$ are centralizers. 
In this paper, we obtain commutativity of $\Gamma$-rings satisfying certain identities involving left centralizer on $\Gamma$-rings. Here we motivated from the works of Ashraf and Ali [8], the results of classical rings are extended to $\Gamma$-rings under the condition (*). Throughout the paper, we consider the $\Gamma$-ring $M$ satisfying (*) $x \beta z \alpha y=x \alpha z \beta y$ for all $x, y$, $z \in M, \alpha, \beta \in \Gamma$. For existence of such a $\Gamma$-ring, we give the following example.

Example: Let $R$ be an associative ring with the unity element 1.Let $M=M_{1,2}(R)$ and $\Gamma=\left\{\left(\begin{array}{c}n .1 \\ 0\end{array}\right): n\right.$ is an integer $\}$. Then $M$ is a $\Gamma$-ring. A simple verification shows that $M$ satisfies the condition (*).

\section{Commutativity of Prime Gamma Rings}

In this section, we have obtained commutativity of $M$.

Theorem 2.1. Let $M$ be a prime $\Gamma$-ring and $I$ be a nonzero ideal of $M$. Suppose that $M$ admits a nonzero left centralizer $T$ such that $T(x) \neq x$, for all $x \in I$. Further, if $T\left([x, y]_{\alpha}\right)-[x$, $y]_{\alpha}=0$, for all $x, y \in I, \alpha \in \Gamma$, then $M$ is commutative.

Proof. Given that $T$ is a left centralizer of $M$ such that

$$
T\left([x, y]_{\alpha}\right)-[x, y]_{\alpha}=0 \text {, for all } x, y \in I, \alpha \in \Gamma \text {. }
$$

This can be rewritten as

$$
(T(x)-x) \alpha y-(T(y)-y) \alpha x=0 \text {, for all } x, y \in I, \alpha \in \Gamma \text {. }
$$

Replacing $x$ by $x \beta r$ in (2), we obtain,

$$
(T(x)-x) \beta r \alpha y-(T(y)-y) \alpha x \beta r=0 \text {, for all } x, y \in I, r \in M, \alpha, \beta \in \Gamma .
$$

Using condition $(*)$ we get,

$(T(x)-x) \beta r \alpha y-(T(y)-y) \beta x \alpha r=0$, for all $x, y \in I, r \in M, \alpha, \beta \in \Gamma$.

Using (2) in (3) to simplify, we obtain

$$
(T(x)-x) \beta[r, y]_{\alpha}=0 \text {, for all } x, y \in I, r \in M, \alpha, \beta \in \Gamma \text {. }
$$

Again, replacing $r$ by $r \delta s$ in (4) and using condition (*) we get from (4), $(T(x)-x) \beta r \delta[s, y]_{\alpha}=0$, for all $x, y \in I, r, s \in M, \alpha, \beta, \delta \in \Gamma$, i.e., $(T(x)-x) \Gamma M \Gamma[s, y]_{\alpha}=\{0\}$, for all $x, y \in I, s \in M, \alpha \in \Gamma$. By the primeness of $M$, we obtain either $[s, y]_{\alpha}=0$ or $T(x)-x=$ 0 , for all $x, y \in I, s \in M, \alpha \in \Gamma$. Since $T(x) \neq x$, we get $[s, y]_{\alpha}=0$, for all $y \in I, s \in M, \alpha \in \Gamma$, and therefore, $I \subseteq Z(M)$. Hence, $M$ is commutative. 
Corollary 2.2. In Theorem 2.1, if a left centralizer is zero, then $M$ is commutative.

Proof. Suppose that $T\left([x, y]_{\alpha}\right)-[x, y]_{\alpha}=0$, for any $x, y \in I, \alpha \in \Gamma$. If $T=0$, then $[x, y]_{\alpha}=0$, for all $x, y \in I, \alpha \in \Gamma$. Therefore, $I$ is commutative. Hence, $M$ is commutative.

Using similar arguments as used in proof of Theorem 2.1, we can prove the following:

Theorem 2.3. Let $M$ be a prime $\Gamma$-ring and $I$ be a nonzero ideal of $M$. Suppose that $M$ admits a nonzero left centralizer $T$ such that $T(x) \neq-x$, for all $x \in I$. Further, if $T\left([x, y]_{\alpha}\right)+$ $[x, y]_{\alpha}=0$, for all $x, y \in I, \alpha \in \Gamma$, then $M$ is commutative.

If we replace the product $[x, y]_{\alpha}$ by $\langle x, y\rangle_{\alpha}$ in Theorems 2.1 and 2.3 , we obtain the following results:

Theorem 2.4. Let $M$ be a prime $\Gamma$-ring and $I$ be a nonzero ideal of $M$. Suppose that $M$ admits a nonzero left centralizer $T$ such that $T(x) \neq x$, for all $x \in I$. Further if $T\left(\langle x, y\rangle_{\alpha}\right)=\langle x$, $y\rangle_{\alpha}$, for all $x, y \in I, \alpha \in \Gamma$, then $M$ is commutative

Proof. Given that

$T\left(\langle x, y\rangle_{\alpha}\right)-\langle x, y\rangle_{\alpha}=0$, for all $x, y \in I, \alpha \in \Gamma$.

This implies that

$(T(x)-x) \alpha y+(T(y)-y) \alpha x=0$, for all $x, y \in I, \alpha \in \Gamma$.

Replacing $x$ by $x \beta r$ in (6), we obtain

$(T(x)-x) \beta r \alpha y+(T(y)-y) \alpha x \beta r=0$, for all $x, y \in I, r \in M, \alpha, \beta \in \Gamma$.

Using (6) and condition (*), we obtain,

$(T(x)-x) \beta r \alpha y-(T(x)-x) \beta y \alpha r=0$, for all $x, y \in I, r \in M, \alpha, \beta \in \Gamma$.

That is,

$(T(x)-x) \beta[r, y]_{\alpha}=0$, for all $x, y \in I, r \in M, \alpha, \beta \in \Gamma$.

Thus, Eq. (9) is same as Eq. (4) and henceforth the proof is follows by the proof of Theorem 2.1.

Proceeding on similarly with necessary variations, we can prove the following:

Theorem 2.5. Let $M$ be a prime $\Gamma$-ring and $I$ be a nonzero ideal of $M$. Suppose that $M$ admits a nonzero left centralizer $T$ such that $T(x) \neq-x$, for all $x \in I$. Further, if $T\left(\langle x, y\rangle_{\alpha}\right)+$ $\langle x, y\rangle_{\alpha}=0$, for all $x, y \in I, \alpha \in \Gamma$, then $M$ is commutative.

Corollary 2.6. In Theorems 2.4 and 2.5 , if a left centralizer $T$ is zero, then $M$ is commutative. 
Proof. For any $x, y \in I, \alpha \in \Gamma$, we have $T\left(\langle x, y\rangle_{\alpha}\right)=\langle x, y\rangle_{\alpha}$. If $T=0$, then $\langle x, y\rangle_{\alpha}=0$, for all $x$, $y \in I, \alpha \in \Gamma$. Replacing $x$ by $x \beta z$ and using the fact that $y \beta x=-x \beta y$, we obtain $x \beta[z, y]_{\alpha}=0$, for all $x, y, z \in I, \alpha, \beta \in \Gamma$, i.e., $I \Gamma M \Gamma[z, y]_{\alpha}=\{0\}$, for all $y, z \in M, \alpha \in \Gamma$. Since $M$ is prime and $I \neq\{0\}$, so that $[z, y]_{\alpha}=0$, for all $y, z \in M, \alpha \in \Gamma$. Thus, $I$ is commutative and hence $M$ is commutative.

Corollary 2.7. Let $M$ be a prime $\Gamma$-ring and $I$ be a nonzero ideal of $M$. Suppose that $M$ admits a nonzero left centralizer $T$ such that $T(x) \neq \pm x$ for all $x \in I$. Further, if $T(x \alpha y) \pm x \alpha y$ $=0$, for all $x, y \in I, \alpha \in \Gamma$, then $M$ is commutative.

Proof For any $x, y \in I, \alpha \in \Gamma$, we have $T(x \alpha y)=x \alpha y$. This implies that $T\left([x, y]_{\alpha}\right)-[x, y]_{\alpha}=$ 0 , for all $x, y \in I, \alpha \in \Gamma$, and hence by Theorem $2.1, M$ is commutative.

On the other hand if $M$ satisfy the condition $T(x \alpha y)+x \alpha y=0$, for all $x, y \in I, \alpha \in \Gamma$. Then for any $x, y \in I, \alpha \in \Gamma$, we have $T(x \alpha y+y \alpha x)=-(x \alpha y+y \alpha x)$. This implies that $T(\langle x$, $\left.y\rangle_{\alpha}\right)+\langle x, y\rangle_{\alpha}=0$, for all $x, y \in I, \alpha \in \Gamma$. Thus, by Theorem 2.5, $M$ is commutative.

Similarly, we can prove the following:

Corollary 2.8. Let $M$ be a prime $\Gamma$-ring and $I$ be a nonzero ideal of $M$. Suppose that $M$ admits a nonzero left centralizer $T$ such that $T(x) \neq \pm x$, for all $x \in I$. Further, if $T(x \alpha y) \pm y \alpha x$ $=0$, for all $x, y \in I, \alpha \in \Gamma$, then $M$ is commutative.

Theorem 2.9. Let $M$ be a prime $\Gamma$-ring and $I$ be a nonzero ideal of $M$. If $M$ admits a nonzero left centralizer $T$ such that $T(x) \neq x$ for all $x \in I$, then the following conditions are equivalent:

(i) $T\left([x, y]_{\alpha}\right)-[x, y]_{\alpha}=0$, for all $x, y \in I, \alpha \in \Gamma$.

(ii) $T\left([x, y]_{\alpha}\right)+[x, y]_{\alpha}=0$, for all $x, y \in I, \alpha \in \Gamma$.

(iii) For all $x, y \in \mathrm{I}, \alpha \in \Gamma$, either $T\left([x, y]_{\alpha}\right)-[x, y]_{\alpha}=0$ or $T\left([x, y]_{\alpha}\right)+[x, y]_{\alpha}=0$.

(iv) $M$ is commutative.

Proof. (iv) $\Rightarrow$ (i), (iv) $\Rightarrow$ (ii) and (iv) $\Rightarrow$ (iii) are obvious. Now, we will prove that (i) $\Rightarrow$ (iv). For any $x, y \in I, \alpha \in \Gamma$, we have $T\left([x, y]_{\alpha}\right)-[x, y]_{\alpha}=0$, then by Theorem $2.1, M$ is commutative. Similarly, we can prove that (ii) $\Rightarrow$ (iv).

(iii) $\Rightarrow$ (iv). For each fixed $y \in I, \alpha \in \Gamma$, we set $I_{1}=\left\{x \in I \mid T\left([x, y]_{\alpha}\right)-[x, y]_{\alpha}=0\right\}$ and $I_{2}=\left\{x \in I \mid T\left([x, y]_{\alpha}\right)+[x, y]_{\alpha}=0\right\}$. Then, $I_{1}$ and $I_{2}$ are additive subgroups of $I$ such that $I=$ $I_{1} \cup I_{2}$. But a group can not be the set-theoretic union of two proper subgroups, hence we 
obtain either $I_{1}=I$ or $I_{2}=I$.. Further, using similar arguments, we obtain $I=\left\{y \in I \mid I_{1}=I\right\}$ or $I=\left\{y \in I \mid I_{2}=I\right\}$. Thus, we obtain that either $T\left([x, y]_{\alpha}\right)-[x, y]_{\alpha}=0$, for all $x, y \in I, \alpha \in \Gamma$ or $T\left([x, y]_{\alpha}\right)+[x, y]_{\alpha}=0$ for all $x, y \in I, \alpha \in \Gamma$. Hence, $M$ is commutative in both the cases by Theorem 2.1. (resp. Theorem 2.3.). The proof of the theorem is complete.

Theorem 2.10. Let $M$ be a prime $\Gamma$-ring and $I$ be a nonzero ideal of $M$. If $M$ admits a generalized derivation $D$ associated with a derivation $d$ such that $D\left([x, y]_{\alpha}\right)-[x, y]_{\alpha}=0$ (resp. $\left.D\left([x, y]_{\alpha}\right)+[x, y]_{\alpha}=0\right)$, for all $x, y \in I, \alpha \in \Gamma$. Moreover, if $D(x) \neq x$, for all $x \in I$, then $M$ is commutative.

Proof. When the associated derivation $d=0$, then using Theorem 2.1, we get the required result. On the other hand if $d \neq 0$, then the proof follows from Theorem 2.1.

Similarly, in view of Theorem 2.4 and 2.5 above, we obtain the following result:

Theorem 2.11. Let $M$ be a prime $\Gamma$-ring and $I$ be a nonzero ideal of $M$. If $M$ admits a generalized derivation $D$ associated with a derivation $d$ such that $D\left(\left(\langle x, y\rangle_{\alpha}\right) \pm\langle x, y\rangle_{\alpha}=0\right.$, for all $x, y \in I, \alpha \in \Gamma$. Moreover, if $D(x) \neq x$, for all $x \in I$, then $M$ is commutative.

Theorem 2.12. Let $M$ be a prime $\Gamma$-ring and $I$ be a nonzero ideal of $M$. Suppose that $M$ admits a nonzero left centralizer $T$ such that $T(x) \neq x$, for all $x \in I$. Further, if $T(x \alpha y)$ $x \alpha y \in Z(M)$, for all $x, y \in I, \alpha \in \Gamma$, then $M$ is commutative.

Proof. For any $x, y \in I, \alpha \in \Gamma$, we have $T(x \alpha y)-x \alpha y \in Z(M)$. This can be rewritten as

$T(x) \alpha y-x \alpha y \in Z(M)$, for all $x, y \in I, \alpha \in \Gamma$.

That is,

$[(T(x)-x) \alpha y, r]_{\beta}=0$, for all $x, y \in I, r \in M, \alpha, \beta \in \Gamma$.

This implies that,

$(T(x)-x) \alpha[y, r]_{\beta}+[T(x)-x, r]_{\beta} \alpha y=0$, for all $x, y \in I, r \in M, \alpha, \beta \in \Gamma$.

Replacing $x$ by $x \delta z$ in (12), we obtain

$(T(x)-x) \delta z \alpha[y, r]_{\beta}+[(T(x)-x) \delta z, r]_{\beta} \alpha y=0$, for all $x, y, z \in I, r \in M, \alpha, \beta, \delta \in \Gamma$.

Combining (11) and (13), we find that $(T(x)-x) \delta z \alpha[y, r]_{\beta}=0$, for all $x, y, z \in I, r \in M$, $\alpha, \beta, \delta \in \Gamma$. This yields that $(T(x)-x) \Gamma M \Gamma I \Gamma[y, r]_{\beta}=\{0\}$, for all $x, y \in I, r \in M, \beta \in \Gamma$. The primeness of $M$ implies that either $I \Gamma[x, r]_{\beta}=\{0\}$ or $T(y)-y=0$, for all $x, y \in I, r \in M, \beta \in \Gamma$. Since $I \neq\{0\}$ and $T(x) \neq x$, for all $x \in I$, we find that $I$ is central and hence $M$ is commutative.

Similar arguments as above yield the following: 
Theorem 2.13 Let $M$ be a prime $\Gamma$-ring and $I$ be a nonzero ideal of $M$. Suppose that $M$ admits a nonzero left centralizer $T$ such that $T(x) \neq-x$, for all $x \in I$. Further, if $T(x \alpha y)+$ $x \alpha y \in Z(M)$, for all $x, y \in I, \alpha \in \Gamma$, then $M$ is commutative.

Proof. Suppose $T$ is a nonzero left centralizer satisfying the property $T(x \alpha y)+x \alpha y \in Z(M)$, for all $x, y \in I, \alpha \in \Gamma$, then the nonzero left centralizer (- $T$ ) also satisfies the condition ($T)(x \alpha y)-x \alpha y \in Z(M)$, for all $x, y \in I, \alpha \in \Gamma$. Hence by Theorem 3.1, $M$ is commutative.

Remark 2.14 In Theorem 2.12, if the left centralizer is zero, then $M$ is commutative.

Theorem 2.15 Let $M$ be a prime $\Gamma$-ring and $I$ be a nonzero ideal of $M$. Suppose that $M$ admits a nonzero left centralizer $T$ such that $T(x) \neq x$, for all $x \in I$. Further, if $T(x \alpha y)$ $y \alpha x \in Z(M)$, for all $x, y \in I, \alpha \in \Gamma$, then $M$ is commutative.

Proof. We are given that $T$ is a left centralizer of $M$ such that $T(x \alpha y)-y \alpha x \in Z(M)$ for all $x$, $y \in I, \alpha \in \Gamma$. This implies that $[T(x) \alpha y-y \alpha x, r]_{\beta}=0$, for all $x, y \in I, r \in M, \alpha, \beta \in \Gamma$. Replacing $y$ by $x \delta y$ in the last relation and using it, we find that $(T(x) \alpha y-y \alpha x) \delta[y, r]_{\beta}=0$, for all $x$, $y \in I, r \in M, \alpha, \beta, \delta \in \Gamma$. Again, replace $r$ by $r \lambda s$ in the above expression, to get $(T(x) \alpha y$ $y \alpha x) \delta r \lambda[y, s]_{\beta}+(T(x) \alpha y-y \alpha x) \delta[y, r]_{\beta} \lambda s=0$, for all $x, y \in I, r, s \in M, \alpha, \beta, \delta, \lambda \in \Gamma$. This yields that $(T(x) \alpha y-y \alpha x) \delta r \lambda[y, s]_{\beta}=0$ i.e., $(T(x) \alpha y-y \alpha x) \Gamma M \Gamma[y, s]_{\beta}=\{0\}$, for all $x, y \in I$, $s \in M, \alpha, \beta \in \Gamma$. The primeness of $M$ implies that either $[y, s]_{\beta}=0$ or $T(x) \alpha y-y \alpha x=0$, for all $x, y \in I, s \in M, \alpha, \beta \in \Gamma$. Now, we put $I_{1}=\left\{x \in I \mid[y, s]_{\beta}=0\right.$ for all $\left.s \in M, \beta \in \Gamma\right\}$ and $I_{2}=$ $\{x \in I \mid T(x) \alpha y-y \alpha x=0$, for all $y \in I, \alpha \in \Gamma\}$. Then, clearly $I_{1}$ and $I_{2}$ are additive subgroups of $I$. Moreover, by the discussion given, $I$ is the set-theoretic union of $I_{1}$ and $I_{2}$. But a group can not be the set-theoretic union of two proper subgroups, hence $I_{1}=I$ or $I_{2}=I$. If $I_{1}=I$, then $[y, s]_{\beta}=0$, for all $y \in I, s \in \mathrm{M}, \beta \in \Gamma$ and hence $M$ is commutative. On the other hand if $I_{2}=I$, then $T(x) \alpha y=y \alpha x$, for all $x, y \in I, \alpha \in \Gamma$. That is $T(x \alpha y)-y \alpha x=0$, for all $x$, $y \in I, \alpha \in \Gamma$. This implies that $T\left([x, y]_{\alpha}\right)-[x, y]_{\alpha}=0$, for all $x, y \in I, \alpha \in \Gamma$. Hence, the application of Theorem 2.1 yields the required result.

Using similar arguments as used in the proof of above theorem, we get the following:

Theorem 2.16 Let $M$ be a prime $\Gamma$-ring and $I$ be a nonzero ideal of $M$. Suppose that $M$ admits a nonzero left centralizer $T$ such that $T(x) \neq-x$, for all $x \in I$. Further, if $T(x \alpha y)+$ $y \alpha x \in Z(M)$, for all $x, y \in I, \alpha \in \Gamma$, then $M$ is commutative. 
Theorem 2.17 Let $M$ be a prime $\Gamma$-ring and $I$ be a nonzero ideal of $M$. If $M$ admits a nonzero left centralizer $T: M \rightarrow M$ such that $T(x) \neq x$, for all $x \in I$. Then the following conditions are equivalent:

(i) For all $x, y \in I, \alpha \in \Gamma$, either $T(x \alpha y)-x \alpha y \in Z(M)$ or $T(x \alpha y)+x \alpha y \in Z(M)$.

(ii) For all $x, y \in I, \alpha \in \Gamma$, either $T(x \alpha y)-y \alpha x \in Z(M)$ or $T(x \alpha y)+y \alpha x \in Z(M)$ for all $x$, $y \in I, \alpha \in \Gamma$.

(iii) $M$ is commutative.

Proof. Obviously, (iii) $\Rightarrow$ (i) \& (ii). Using similar techniques, as used to prove (iii) $\Rightarrow$ (iv) in case of Theorem 2.9, it can be easily shown that (i) $\Rightarrow$ (iii) and (ii) $\Rightarrow$ (iii).

Theorem 2.18 Let $M$ be a prime $\Gamma$-ring and $I$ be a nonzero ideal of $M$. Suppose that $M$ admits a generalized derivation $D$ associated with a derivation $d$ and a nonzero left centralizer $T$ such that $T(x) \neq x$, for all $x \in I$. Further, if $D(x \alpha y)-x \alpha y \in Z(M)$, for all $x, y \in I$, $\alpha \in \Gamma$, then $M$ is commutative.

Proof. Combining the proof of Theorem 2.12 above and Theorem 2.1 of [7], we get the required result.

Theorem2.19 Let $M$ be a prime $\Gamma$-ring and I be a nonzero ideal of $M$. Suppose that $M$ admits a generalized derivation $D$ associated with a derivation $d$ and a nonzero left centralizer $T$ such that $T(x) \neq x$, for all $x \in I$. Further, if $D(x \alpha y)-y \alpha x \in Z(M)$, for all $x, y \in I$, $\alpha \in \Gamma$, then $M$ is commutative.

Remark 2.20 It can be easily seen that the above results which are obtained for left centralizers are also true in the case of right centralizers.

\section{Acknowledgement}

The authors are greatfull to the referee for his/her usefulcomments and suggestions.

\section{References}

1. W. E. Barnes, Pacific J. Math. 18, 411 (1966). http://dx.doi.org/10.2140/pim.1966.18.411

2. E. C. Posner, Proc. Amer. Math. Soc. 8, 1093 (1957). http://dx.doi.org/10.1090/S0002-9939-1957-0095863-0

3. M. Bresar and J. Vukman, Proc. Amer. Math. Soc. 110, 7 (1990).

4. M. Asci and S. Ceran, Int. Math. Forum 2, 103 (2007).

5. M. Soyturk, Turk. J. Math. 18, 149 (1999).

6. M. F. Hoque and A. C Paul, Inter. Math. Forum 6 (13), 627 (2011).

7. K. K. Dey and A. C. Paul, J. Sci. Res. 4 (2), 349 (2012). http://dx.doi.org/10.3329/jsr.v4i2.8691 
8. M. Ashraf and S. Ali, Demonstratio Mathematica 41 (4), 763 (2008).

9. H. E. Bell and W. S. Martindale III, Canad. Math. Bull. 30, 92 (1987). http://dx.doi.org/10.4153/CMB-1987-014-x

10. M. A. Quadri, M. S. Khan and N. Rehman, Indian J. Pure Appl. Math. 34 (9), 1393 (2003).

11. J. Vukman, Comment. Math. Univ. Carolinae 42, 237 (2001).

12. J. Vukman, Comment. Math. Univ. Carolinae 40, 447(1999).

13. B. Zalar, Comment. Math. Univ. Carolinae 32, 609 (1991).

14. Q. Deng and M. Ashraf, Results in Math. 30, 259 (1996). http://dx.doi.org/10.1007/BF03322194

15. J. H. Mayne, Canad. Math. Bull. 27, 122 (1984). http://dx.doi.org/10.4153/CMB-1984-018-2 